\title{
Circadian rhythms and renal pathophysiology
}

\author{
Rajesh Mohandas, ${ }^{1,2}$ Lauren G. Douma, ${ }^{3}$ Yogesh Scindia, ${ }^{1,4,5}$ and Michelle L. Gumz ${ }^{1,2,3,6}$ \\ 'Department of Medicine, Division of Nephrology, ${ }^{2}$ Center for Integrative Cardiovascular and Metabolic Diseases, ${ }^{3}$ Department of Biochemistry and Molecular Biology, ${ }^{4}$ Department of Medicine, Division of \\ Pulmonary, Critical Care, and Sleep Medicine, ${ }^{5}$ Department of Pathology, and ${ }^{6}$ Department of Physiology and Functional Cenomics, University of Florida, Cainesville, Florida, USA.
}

\begin{abstract}
The reality of life in modern times is that our internal circadian rhythms are often out of alignment with the light/dark cycle of the external environment. This is known as circadian disruption, and a wealth of epidemiological evidence shows that it is associated with an increased risk for cardiovascular disease. Cardiovascular disease remains the top cause of death in the United States, and kidney disease in particular is a tremendous public health burden that contributes to cardiovascular deaths. There is an urgent need for new treatments for kidney disease; circadian rhythm-based therapies may be of potential benefit. The goal of this Review is to summarize the existing data that demonstrate a connection between circadian rhythm disruption and renal impairment in humans. Specifically, we will focus on chronic kidney disease, lupus nephritis, hypertension, and aging. Importantly, the relationship between circadian dysfunction and pathophysiology is thought to be bidirectional. Here we discuss the gaps in our knowledge of the mechanisms underlying circadian dysfunction in diseases of the kidney. Finally, we provide a brief overview of potential circadian rhythm-based interventions that could provide benefit in renal disease.
\end{abstract}

\section{Introduction}

Renal function is essential to maintaining systemic homeostasis. The kidneys filter the blood, maintain body $\mathrm{pH}$, water, and electrolyte balance, and contribute to the regulation of blood pressure. The kidneys produce urine as a byproduct of maintaining fluid and electrolyte balance. The volume and composition of the urine can provide insight into health status. Indeed, analysis of the urine is thought to be the oldest medical test, dating back to the ancient Babylonians and Sumerians who recorded their observations on clay tablets more than 6000 years ago (1). This practice was termed "uroscopy," meaning the scientific examination of urine. Thousands of years later, Hippocrates practiced uroscopy and believed that changes in the properties of urine were indicative of disease states. For example, from this time through the Middle Ages, medical practitioners would taste urine in order to detect sweetness, which was indicative of diabetes mellitus. In the 11th century, the physician Ismail al-Jurjani noted that urine composition could be altered by food intake as well as by aging. Interestingly, Ismail purportedly asked for a full 24-hour collection of urine and emphasized the importance of a good night's sleep prior to the collection. This report provides perhaps the earliest hint at the incorporation of time as a biological variable in the practice of medicine.

It was documented as early as the end of the 19th century (reviewed in ref. 2) that urine volume varies over the course of a 24-hour period, although this observation was largely attributed to differences in fluid intake in the active versus the rest phase. In the

Conflict of interest: YS is an inventor on US patent application no. 16/597,634 ("Modulating iron metabolism delays the on-set and severity of lupus nephritis").

Copyright: $\odot$ 2022, Mohandas et al. This is an open access article published under the terms of the Creative Commons Attribution 4.0 International License.

Reference information: J Clin Invest. 2022;132(3):e148277.

https://doi.org/10.1172/JCl148277. 1950s, landmark studies by Mills and colleagues firmly established the rhythmicity of renal function (3-5). These investigators showed that urinary sodium, potassium, chloride, and phosphate were excreted with a 24-hour rhythmic pattern and that urine volume and $\mathrm{pH}$ varied over the 24-hour cycle as well. Importantly, these experiments were conducted under relatively constant conditions so that fluid and food intake, posture, and sleep stage were all controlled. Thus, it could be concluded that the rhythmic variations were endogenous and not a result of behavior or posture. In the decades since, enormous strides have been made in understanding the molecular mechanisms underlying rhythmic renal function. The discovery of circadian clock genes, for which Hall, Rosbash, and Young were awarded the Nobel Prize in 2017, made it possible to study the molecular mechanisms of circadian rhythms (6).

The circadian system is conducted by the central clock, located in the suprachiasmatic nucleus of the hypothalamus, through neuronal and humoral signaling to the "orchestra" of the body's peripheral clocks, which include other regions of the brain and tissue clocks such as the kidney. Indeed, the molecular machinery of the circadian clock is present in nearly every cell and tissue type in humans, and its mechanisms have been extensively reviewed (7, 8). Briefly, the core components of the clock comprise transcription factors that function in a transcription-translation feedback loop (Figure 1). In the positive arm, BMAL1 and CLOCK proteins heterodimerize and bind E-box response elements in the promoters of target genes. These target genes include those encoding the PER and CRY proteins that function in the negative arm of the feedback system. PER and CRY feed back on and inhibit the activity of BMAL1 and CLOCK, thus decreasing the transcription of their own genes in addition to other target genes. This mechanism contributes to the regulation of nearly half of all expressed genes in a tissue-specific manner and plays a major role in rhythmic physiological function, including blood pressure rhythms and 


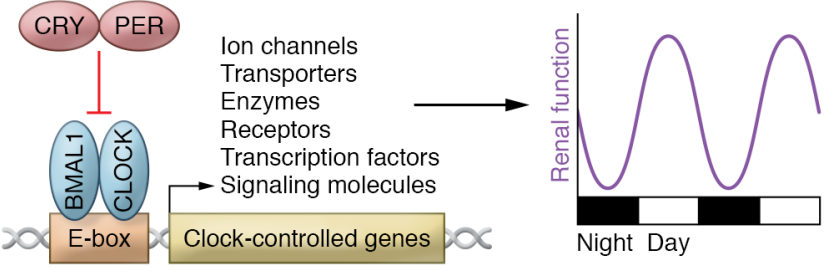

Figure 1. Transcription-translation feedback loop of the circadian clock. BMAL1 and CLOCK bind to E-box response elements in the promoters of target genes, which include Period and Cryptochrome. PER and CRY form the negative arm of this feedback loop. Ancillary loops of the transcription-translation feedback system involving nuclear receptors and posttranslational modifications exist but will not be discussed here. Also beyond the scope of this Review is a discussion of the non-canonical functions of clock proteins, such as the role of BMAL1 in the regulation of translation in the cytosol (161)

renal excretion rhythms (refs. 9-17 and Figure 1). In the kidney, for example, animal studies have demonstrated that genes encoding proteins involved in xenobiotic metabolism as well as various sodium transport genes are regulated by the clock mechanism (18-20). In humans, a small study in healthy volunteers showed that protein levels of $\mathrm{Na}^{+}-\mathrm{Cl}^{-}$cotransporter (NCC) and prostasin in urinary exosomes varied over a 24 -hour period (21), consistent with what has been shown using mouse models.

The circadian system is thought to have evolved in order to provide an adaptive homeostatic advantage for organisms living on a planet with 24-hour periods of light and dark. The molecular clock components are highly conserved: humans share approximately $30 \%$ homology with the BMAL1 homolog in bread mold, for example (22). Given the 24-hour, 7-days-a-week nature of modern life, disruption of the circadian system is increasingly common (23). Circadian disruption is defined as misalignment between the endogenous circadian rhythms of our internal body clocks and the external environment. Circadian disruption can occur acutely: a shift of just 1 hour due to daylight saving time is associated with an increase in adverse cardiovascular events (24). Epidemiological studies in this century have demonstrated that chronic circadian disruption, such as what occurs in shift workers, is a key risk factor for a number of pathologies, including cancer and cardiovascular disease. For example, shift workers have an increased risk of developing chronic kidney disease (25) and hypertension (26). Long working hours can cause circadian disruption and are associated with declining renal function (27). A recent meta-analysis demonstrated a link between long-term night shift work and increased systolic blood pressure (28). Sleep disorders, such as obstructive sleep apnea, are associated with both hypertension and non-dipping hypertension (29). There also seems to be a genetic relationship between single-nucleotide polymorphisms (SNPs) in circadian clock genes and the prevalence of some diseases. A genome-wide association study of 1304 individuals across 424 British families revealed BMAL1 haplotypes that are associated with the hypertension phenotype (30). SNPs in clock genes are associated with phenotypic variance in systolic blood pressure (31).

In this Review, we describe evidence linking circadian disruption to the pathologies of chronic kidney disease, lupus nephritis, aging, and hypertension. We also discuss the limitations of our understanding of the molecular mechanisms underlying these pathological states. It is important to note that the relationship between circadian disruption and disease states is bidirectional. In some cases, we do not know what comes first, the circadian malfunction or the disease state. However, we do know that circadian disruption and disease states constitute a vicious cycle in which each condition exacerbates the other. Finally, we discuss possible circadian-based interventions that might provide a benefit for renal function.

\section{Circadian disruption in diseases of the human kidney}

Chronic kidney disease. Chronic kidney disease (CKD) is defined as damage to the kidneys or decrease in renal function that is sustained over at least 3 months. CKD is associated with disordered circadian rhythms of sleep, blood pressure, and proteinuria. In human subjects, worsening renal function is associated with later onset, shorter duration, and increased fragmentation of sleep (32). Consistent with these effects on sleep being due to kidney disease per se, rather than the effect of comorbidities, animal models of kidney disease, including subtotal nephrectomy in rats (33) and adenine model in mice (34), demonstrate abnormalities in sleep and activity. On the other hand, in patients with type 1 diabetes, circadian disruption precedes microalbuminuria or other clinical evidence of kidney disease (35). Similarly, mice with genetic deletion of the circadian CLOCK protein are more vulnerable to adenine-induced CKD than wild-type mice (34). These observations suggest that circadian dysfunction might predispose to kidney disease. Thus, CKD and circadian dysfunction might have a bidirectional relationship, with circadian disruption accelerating kidney dysfunction and kidney disease causing abnormalities in circadian rhythms.

CKD is also associated with non-dipping pattern of blood pressure, suggesting disrupted circadian rhythms of blood pressure. Clinically, non-dipping is defined as less than $10 \%$ difference between night and day blood pressure. The prevalence of non-dipping hypertension in CKD varies from $60 \%$ to $80 \%$ and increases with worsening kidney function (36). In patients with CKD, non-dipping pattern of blood pressure is independently associated with an increased risk of death or progression to endstage renal disease (37). In a small study of 20 patients with endstage renal disease who received kidney transplantation and had ambulatory blood pressure monitoring performed before and a year after the transplant, the percentage of patients who were considered dippers rose from $15 \%$ at 1 month before transplantation to almost $40 \%$ a year after transplantation (38). The dipping status was influenced by the renal function after transplantation and the use of immunosuppressive medications. Although the data on whether nighttime dosing of antihypertensive medication reduces nocturnal blood pressure or restores nocturnal dipping are inconclusive (39-41), a randomized controlled trial of 661 patients with CKD, in which subjects were randomized to receive at least one of their antihypertensive medications at night, found that the risk of cardiovascular events was one-third in comparison with those who received all their medications in the morning (42). The reduction in cardiovascular events seemed out of proportion to that expected from the modest decrease in 
A
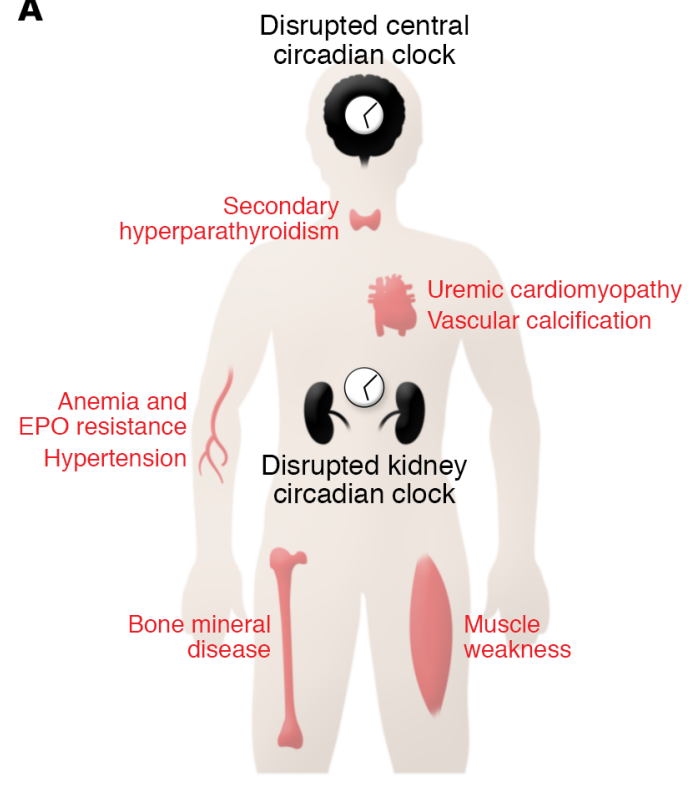

B

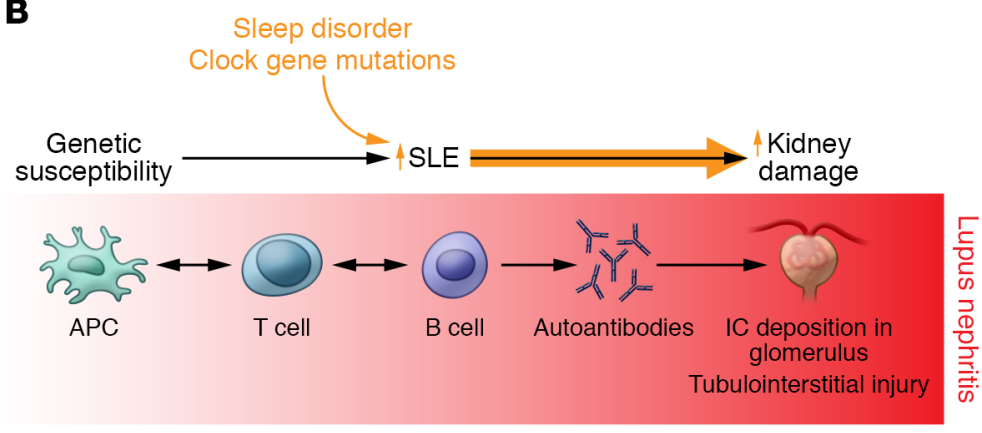

Figure 2. Disruption of circadian rhythms in disease state. (A) Complications of CKD. Kidney disease is associated with disruption of peripheral and central circadian rhythms. The molecular clock modulates the levels or activity of serum phosphate, parathyroid hormone, erythropoietin (EPO), and other hormones that are known to exhibit diurnal rhythms. (B) Schema of progression of SLE to end-organ renal disease (lupus nephritis [LN]) and potential contribution from disturbed circadian clock. Genetically susceptible individuals develop SLE. During disease progression there is a complex crosstalk between multiple cell types involving both innate and adaptive immune systems. The antigen-presenting cells (APCs) present self-antigens from various sources to T lymphocytes, which results in generation of autoreactive T cells. These CD4+ T lymphocytes in turn instruct B cells to produce autoantibodies of different specificities that deposit as immune complexes (ICs) in the glomeruli. This leads to progressive glomerular pathology and local production of chemoattractants and matrix proteins, resulting in immune cell infiltration and tissue damage. Loss of glomerular permeability also leads to tubulointerstitial injury, which is perpetuated by intrinsic tubular cell inflammatory phenotype and infiltrating immune cells and eventually leads to renal failure. Sleep fragmentation or genetic mutations in key clock proteins in SLE patients can potentially accentuate immune cell effector function. Furthermore, mutations in the renal intrinsic cells' clock genes can render them susceptible to injury, as local injurious events unfold during the progression of LN.

blood pressure, suggesting the possibility that restoration of circadian rhythms could have blood pressure-independent effects on the cardiovascular system.

The renin-angiotensin-aldosterone system is upregulated in CKD. Angiotensin II acting via its cognate receptor AT1 modulates the activity of the suprachiasmatic neurons in vitro (43). Whether AT1 activation modulates the central circadian clocks in vivo and contributes to circadian dysfunction in CKD is unknown. Further, gut dysbiosis and accumulation of uremic toxins in CKD might disrupt the circadian clock. The aryl hydrocarbon receptor, an environmental sensor that can bind uremic toxins, demonstrates rhythmic expression and forms a heterodimer with BMAL1 to modulate expression of circadian genes $(44,45)$. However, whether the aryl hydrocarbon receptor contributes to circadian dysfunction in CKD remains to be determined.

CKD is associated with the development of long-term complications such as hyperphosphatemia, secondary hyperparathyroidism, vascular calcification, accelerated atherosclerosis, bone mineral disorders, and anemia. Serum phosphate levels exhibit a circadian rhythm in healthy individuals (46). While the afternoon peak is attenuated by a low-phosphate diet, the nocturnal peak appears to be independent of diet and modulated by unidentified endogenous factors. This raises the intriguing possibility that the circadian system might regulate serum phosphate levels. Although reports of altered rhythmicity of serum phosphate with $\mathrm{CKD}$ in human subjects are variable, a recent study of mice with adenine-induced kidney disease suggests that circadian rhythmicity of phosphate is disrupted only by a high-phosphate diet and induction of vascular calcification (47). However, the molecular mechanisms underlying these observations remain to be elucidated, and whether circadian disruption can lead to vascular calcification needs further study. Similarly, circadian rhythmicity has been observed in the levels or activity of most hormones involved in bone mineral metabolism (48, 49). Erythropoietin levels (50), reticulocyte counts (51), and parathyroid hormone (52) all exhibit circadian rhythmicity in human subjects. However, the role of circadian dysregulation in contributing to the development of long-term complications of CKD remains to be studied.

The signaling mechanisms that are perturbed in CKD can conceivably disrupt the kidney clock. However, disrupted sleep in human subjects with CKD and experimental evidence for disturbed activity and central clock output from animal studies discussed earlier suggest that the central clock in the suprachiasmatic nucleus is perturbed in kidney disease as well. This circadian dysfunction is likely to exacerbate the known complications of CKD (Figure 2A). Although the evidence for circadian dysfunction in CKD is compelling, the molecular mechanisms involved in the pathogenesis remain largely unknown.

Lupus nephritis. Although sleep disruption and fatigue are prominent complaints in patients with systemic lupus erythematosus (SLE) and negatively affect quality of life (53-57), the role 
of the circadian clock in SLE and lupus nephritis (LN), a common complication in patients with SLE, has not been explored in detail. We briefly discuss the general pathobiology of LN and summarize the current knowledge linking disturbances in clock cycle and SLE.

SLE is an autoimmune disease of unknown etiology that mainly affects women of reproductive age. LN is the most common end-organ manifestation of SLE and is the major cause of morbidity and mortality $(58,59)$. Immune complex deposits, composed of antinuclear, anti-C1q, and cross-reactive antiglomerular autoantibodies $(60-64)$, are found in the glomeruli $(65,66)$ and are considered the most common initiators of renal disease in SLE. This leads to local production of cytokines and chemokines that recruit leukocytes to perpetuate renal injury (67-69). The T and B lymphocytes from LN kidneys are clonally expanded, and the same $\mathrm{T}$ cell clones have been detected in the peripheral blood $(70,71)$. Macrophages infiltrate LN kidneys, and this is associated with poor outcomes (72-74). These intrarenal innate and adaptive immune responses can synergize with systemic autoimmunity and worsen overall outcomes. Although the circadian clock has been shown to regulate immune responses and macrophage function, the role of the circadian system in the development of SLE or LN has not been explored in detail.

Sleep disturbances are commonly reported in SLE patients (56, $75,76)$ and negatively affect quality of life (57). Early studies revealed that in patients with SLE, levels of circulating immune complexes drop during sleep, supporting a role for the circadian system in the regulation of immunological processes in SLE (77). The association between sleep quantity and incidence of SLE (78) was studied in a cohort of individuals at increased genetic risk for SLE (79) who did not meet diagnostic criteria for SLE (80) at their baseline visit. In a follow-up study using questionnaires and chart review, less than 7 hours of sleep per night was independently associated with increased risk of developing SLE, suggesting a possible causal role for sleep disturbances and perhaps circadian dysfunction in pathogenesis of SLE (78). In patients with chronic glomerulonephritis and SLE who were treated with the glucocorticoid prednisolone, the nighttime dip in blood pressure was lost, with the blood pressure lowest in the afternoon, rising throughout the night, and peaking in the morning (81). Additional studies are needed to evaluate the role of this circadian disruption in disease progression.

Dysregulated immune responses are a cardinal feature of SLE/ LN, and immune cells display a circadian effector pattern. Perturbations in immune cell circadian clock can influence outcomes of SLE/LN. In humans, natural regulatory T cell (Treg) number and function follow a rhythm across a 24 -hour period. Sleep deprivation abrogated this rhythm and impaired Treg function (82). These findings are noteworthy, as Tregs are dysregulated and functionally impaired in SLE $(83,84)$, raising the possibility that alterations in the circadian clock in SLE could impair Treg functions.

Macrophages are the harbingers of renal disease in LN and have been implicated in the pathology of several spontaneous and induced models of murine $\operatorname{LN}(73,85-87)$. It is well documented that a circadian clock in macrophages controls their inflammatory responses as well as mitochondrial metabolism (88-91). The circadian clock protein BMAL1 regulates IL-1 $\beta$ in macrophages via nuclear factor erythroid 2-related factor 2 (Nrf2) (90). Nrf2 expression is increased in glomeruli of patients with $\mathrm{LN}$, and in mouse models of LN Nrf2 suppresses LN through inhibition of oxidative injury and the NF- $\kappa \mathrm{B}-$ mediated inflammatory response (92). Similarly, BMAL1-knockout macrophages are unable to sustain mitochondrial function, and display enhanced glycolysis as well as HIF-1 $\alpha-$ dependent metabolic reprogramming and inflammatory response (89). In an animal model of $\mathrm{LN}$, renal macrophages switch toward glycolysis, secrete more IL-1 $\beta$, and recruit neutrophils to damage the kidneys (93). Collectively, these findings pose several pertinent questions, like how local microenvironment dictates the function of macrophage molecular clock, particularly in LN.

Few studies have examined the effect of genetic deletion of clock proteins in mice with spontaneous or drug-induced lupus. Mice deficient in the circadian clock genes $C r y 1$ and $C r y 2$ have elevated serum IgG concentrations, serum antinuclear antibodies, and precipitation of $\operatorname{IgG}$, IgM, and complement in glomeruli (94). This study also showed that CRY deficiencies enhance the rate of B cell maturation and stimulate B cell developmental in the spleen and peritoneal cavity, leading to an increase in autoantibody production. Another study, by Palma et al., showed that NZB/W mice (spontaneous model of LN) subjected to sleep deprivation exhibited an earlier onset of the disease, as reflected by the increased number of antinuclear antibodies, though the severity of the disease based on proteinuria and survival data was comparable (95). In contrast, using the same strain of mice, Mishra et al. recently reported significant differences in the renal expression of circadian clock-associated genes and proteins in young and nephritic mice (96). The kidneys of young NZB/W mice displayed a normal circadian pattern of expression of Bmal1, Clock, Per, and Cry genes compared with the nephritic mice. There was also a significant reduction in BMAL1 protein expression at various time points in nephritic NZB/W mice compared with young mice.

The finding that the disturbances in circadian rhythm and dysregulated expression of key clock genes and proteins are associated with progression of SLE and LN is becoming well documented. Collectively, genetic mutations and sleep disturbance may synergize with systemic and local inflammation to worsen LN (Figure 2B). Nevertheless, there are limited reports regarding the role of the circadian clock in progression of LN or other end-organ pathology associated with SLE. Given the known link between activation of the proinflammatory transcription factor NF- $\mathrm{B}$ and lupus (97), it is interesting to note that NF- $\kappa \mathrm{B}$ has been linked to circadian disruption and altered clock gene expression in response to inflammation (98). A recent report showed that circadian disruption during inflammation could be ameliorated by NF- $\mathrm{B}$ inhibition in a mouse model of obesity (99), raising the question of whether NF- $\kappa$ B inhibition could affect circadian disruption in LN. Whether disruptions in circadian rhythm affect SLE manifestations and whether the circadian clock is a target for treatment of lupus and its associated morbidities remain to be determined.

Hypertension. Because the kidneys play a critical role in blood pressure regulation, hypertension can be both cause and consequence of renal damage. Circadian disruption is likely to exacerbate these effects since it is known to negatively affect human health. Shift work is becoming more common in the 21st century (28). It is estimated that about $20 \%$ of workers in Europe and the United States have a shift work schedule, defined as something 
other than a " 9 to 5 " schedule. Shift work is associated with various adverse health effects, including increased prevalence of hypertension. A meta-analysis of 45 studies, including a total of 117,252 workers, found that there was a significant increase in both systolic and diastolic blood pressure in permanent night workers (28). Rotational shift work was associated with a significant increase in systolic blood pressure only. There was no significant association between shift work and hypertension in this study, but the average worker age was below 40 years old. Data from Korea showed that among factory workers taking antihypertensive medications, blood pressure control was worse in night shift workers compared with day shift workers (OR 0.74, 95\% CI 0.68-0.80; ref. 100). This finding remained significant even after adjustment for age, sex, obesity, exercise, smoker status, and alcohol intake. Studies like these demonstrate the risks associated with disruption of normal circadian patterns of behavior and physiology.

Many patients diagnosed with essential hypertension have a normal day/night pattern of blood pressure rhythms, but the overall mean arterial pressure is shifted up. Hypertensive patients with a reduced day/night rhythm of blood pressure are classified as having non-dipping hypertension. The prevalence of non-dipping hypertension is difficult to estimate since it requires the use of 24-hour ambulatory blood pressure monitoring. It is of paramount importance to increase the use of this method, however, as non-dipping hypertension is a critical risk factor for adverse cardiovascular outcomes (reviewed in ref. 101). Early studies demonstrated that restricting dietary sodium could restore the nocturnal dip of individuals with sodium-sensitive blood pressure (102). Patients with essential hypertension and an intact blood pressure dip at night maintain urinary sodium excretion rhythms on a highsalt diet, whereas patients with a non-dipping phenotype lose their rhythms of urinary sodium excretion (103). Sodium restriction significantly improved the rhythms of both urinary sodium excretion and blood pressure in the non-dipping hypertension group, but had no effect on the dipper group.

Timed urine collections provide an opportunity to assess circadian disruption in a noninvasive manner. The loss of the nighttime dip in blood pressure has been linked to disruption of rhythms in urinary sodium excretion (103). It should be noted that a recent study in mice showed that the blood pressure rhythm could be inverted by restriction of food intake to the inactive period, and this effect occurred in the absence of changes in the diurnal pattern of urinary sodium excretion (104). In a pathophysiological setting, it was recently shown that restricting food intake to the active period restored the normal blood pressure rhythm in non-dipping, diabetic mice (105). In a clinical study, 642 Chinese adults with primary hypertension underwent 24-hour ambulatory blood pressure monitoring, and 24-hour urine sodium excretion was measured along with morning urine sodium concentration (106). Both 24-hour urinary sodium excretion and morning urinary sodium concentration had a positive association with blood pressure. Healthy individuals excrete more sodium during the day than during the night. In a cross-sectional study with over 1000 patients in Switzerland, Del Giorno et al. paired 24-hour ambulatory blood pressure data with night and day urine collections to assess the relationship between blood pressure dipping, day/night ratios in sodium excretion, and age (107). Patients with the lowest ratio of day/night sodium excre- tion tended to be older ( $>50$ years of age) and have higher nighttime blood pressure (5-6 mmHg). These findings support the idea that the loss of renal excretion rhythms is associated with aging and may negatively affect blood pressure dipping. This study clearly demonstrates the utility of using timed urine collections as a way to assess rhythmic renal function. Given the strong association between renal sodium excretion and blood pressure, this method could also provide a means to identify those at risk for hypertension-associated end-organ damage.

Aging. It is well established that kidney function decreases with increasing age (108-110). Age-related renal structural changes are observed independent of comorbidities. Kidney mass declines after age 50, with an accompanying decline in the number of functional glomeruli and nephrons (111-113). The incidence of both nephrosclerosis and glomerulosclerosis is significantly higher in patients above 70 years old, even when examining biopsies from normotensive individuals (114-117). The structural changes that occur in the aging kidney are accompanied by alterations of renal physiology. Glomerular filtration rate (GFR) declines with increasing age, but the rate at which GFR decreases with age is still uncertain, as different studies report varying degrees of GFR decline (111,118-122). A decrease in renal blood flow is also observed with increasing age, which is also associated with an increase in renal vascular resistance and filtration fraction $(123,124)$. Dysregulation of electrolyte handling has also been observed among the elderly. Older adults have reduced lithium clearance compared with young subjects, despite the fact that the sodium excretion is similar (123). This suggests that proximal tubule reabsorption of sodium seems to increase with age, but distal nephron sodium reabsorption decreases, which could contribute to the higher rate of salt-sensitive blood pressure among the elderly $(125,126)$.

In addition to overall changes in kidney structure and function, circadian rhythms in behavior, sleep/wake cycle, and other physiological parameters are negatively affected in older populations (127). Changes in sleeping patterns are extremely common among aging adults (128). As age increases, the sleep/ wake cycle shifts and individuals tend to fall asleep and wake up earlier than younger individuals $(129,130)$. Cortisol, which has been demonstrated to regulate rhythms in peripheral clocks, has an altered rhythm of production in older adults $(131,132)$. Nighttime cortisol seems to increase with age in both men and women, but an increase in the morning acrophase of cortisol is observed only in women (133). These changes in cortisol are likely related to the age-related sleep cycle changes, but may also affect renal rhythms. Multiple studies in varying aging populations have demonstrated that renal diurnal rhythms deteriorate with age. Electrolyte excretion rhythms have been shown to dampen with increasing age (134). Compared with patients in the 25 to 35 age range, healthy subjects in the 60- to 80-year-old group had a reduced day-to-night ratio of water, electrolyte, and solute excretion (135). Additionally, older individuals showed reduced 24-hour sodium and potassium excretion, despite solute excretion and urine volume similar to those in the younger group.

A common symptom associated with aging is nocturia, which is defined as waking up one or more times at night to urinate (136). The prevalence of nocturia increases with age in both men and 


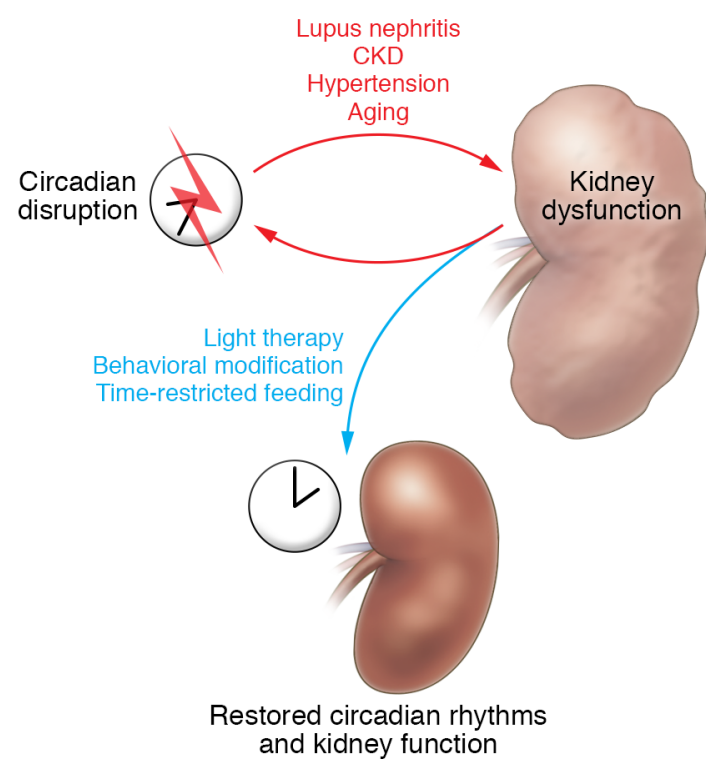

Figure 3. Proposed reciprocal relationship between circadian disruption and kidney disease. Circadian disruption may lead to renal disease, but kidney disease itself may cause circadian disruption. These pathological states may exacerbate each other. This vicious cycle may represent an opportunity for circadian rhythm-based interventions as novel therapies to restore circadian rhythms and physiological function.

women, with around $60 \%$ of people older than 70 waking up two or more times nightly to urinate $(137,138)$. Nocturia pathophysiology in the elderly involves many different factors, but significant contributions arise from age-related changes in the kidney, such as reduced ability to concentrate urine (139) and the inability to excrete solutes shortly after a meal (140). Compared with agematched non-nocturic patients, older nocturic patients not only had larger nighttime urine volume, but also excreted significantly more sodium, chloride, and potassium at night $(141,142)$. The nocturic patients excreted half of their total 24-hour sodium within the nighttime period, whereas the non-nocturic patients excreted twice the amount of sodium during the daytime compared with the nighttime. No differences were observed in urine aldosterone excretion rates between the two groups. Furthermore, decreased day-to-night ratios (altered diurnal rhythms) of diuresis in older men with nocturia are associated with higher nighttime mean arterial pressure (143). The alteration of circadian rhythms in patients with nocturia is associated with sleep disturbances, depression, arterial hypertension, and increased mortality and morbidity $(144,145)$. Despite the negative effects of nocturia, often patients do not report their symptoms to their physician because they consider nocturia to be part of the normal aging process (146).

Clinically, it is critical to determine the mechanisms behind dysregulation of the circadian clock in the aging kidney to improve quality of life and treatment strategies. For example, in a retrospective study using the US Renal Data System Dialysis Morbidity and Mortality Waves III/IV database, researchers examined the impact of the time of day at which dialysis was administered in a population of patients older than 60 years (147). Elderly patients who underwent dialysis in the morning or in the evening had a lower risk for mortality compared with those who received dialysis in the afternoon. Interestingly, time of day of dialysis did not seem to have an effect on mortality in patients younger than 60 . These results are similar to those of an earlier study that reported increased survival of elderly end-stage renal disease patients who underwent dialysis in the morning versus the afternoon (148). Interventions and lifestyle changes such as exercise (149) have been hypothesized to help maintain renal rhythms, but this is a poorly researched area that could greatly benefit from additional studies. Additionally, the mechanisms that contribute to altered circadian rhythms in the elderly are still poorly understood and warrant further research. DNA methylation and histone modifications change with age, primarily in response to environmental stimuli as shown by studies with monozygous twins (150). These epigenetic changes may contribute to altered circadian rhythms in the elderly.

\section{Circadian therapeutics and the future}

It is well established that loss of circadian rhythms in physiological functions can lead to disease. The evidence that this phenomenon occurs in patients with kidney disease is growing. As basic research brings us closer to understanding the pathophysiological mechanisms by which circadian disruption causes disease, the potential for circadian rhythm-based interventions is increasing. Using timed light therapy as an intervention, one small randomized controlled trial found that night shift workers had improved nighttime blood pressure dip with treatment. This increase in the nighttime blood pressure dipping status was associated with a decrease in serum glucose during oral glucose tolerance testing. There were no changes in serum insulin, melatonin, or cortisol, but plasma catecholamine levels were reduced (151). Early time-restricted feeding (all meals before $3 \mathrm{pm}$ ) in prediabetic men lowered blood pressure and increased insulin sensitivity (152). In a study including both male and female patients, a 10-hour self-selected window for eating resulted in lower blood pressure as well as a reduction in hemoglobin $A_{1 c}$ (153). In terms of treating patients with endstage kidney disease, home dialysis and nocturnal dialysis offer attractive options for improving quality of life and potentially survival $(154,155)$. Whether some of the benefits are due in part to restoration of circadian rhythms is an intriguing possibility that merits further study. Preclinical rodent studies suggest that circadian clock modulatory compounds may have benefit in cardiovascular disease (156). There is growing interest in "drugging the clock," which could include optimizing the timing of medication delivery as well as small molecules that influence clock protein function, in humans in several different diseases (157). With the increasing availability of circadian rhythm-based therapeutics, it is becoming more and more important that we gain a more complete understanding of the mechanisms by which the circadian clock system regulates physiological function and how circadian disruption contributes to pathophysiology.

In the future, adoption of circadian-based therapies will require development of tests to assay the extent of circadian disruption to facilitate diagnosis and assess response to therapy. The use of timed urine collections could shed light on the incidence of circadian disruption and help assess the effectiveness of circadian-based therapies in a noninvasive manner. Measurement of 
clock gene expression in blood samples may also be useful as a prognostic and diagnostic biomarker in patients on dialysis, those with CKD, or those with or at risk for acute kidney injury (158-160). Although the potential to use circadian principles in the treatment or management of kidney disease is growing (Figure 3), the mechanisms by which disrupted circadian rhythm mediates and perpetuates renal injury remain to be elucidated, as basic research in this area is still in the early stages. Given the physiological and clinical significance of circadian rhythms to human health, more detailed examination of the circadian clock in models of renal disease is necessary and is expected to bring a deeper understanding of renal versus systemic circadian homeostasis.

\section{Acknowledgments}

This work was supported by the National Institute of Diabetes and Digestive and Kidney Diseases grant R01DK109570 (to MLG), American Heart Association Established Investigator Award (to MLG), NIH grant K08HL130945 (to RM), Florida Department of Health James and Esther King Award (to MLG and RM), and Vifor Pharma grants P0213104 and P0226952 (to YS).

Address correspondence to: Michelle L. Gumz, Department of Physiology and Functional Genomics, University of Florida, 1345 Center Drive, Box 100274, Gainesville, Florida 32610, USA. Phone: 352.273.6887; Email: Michelle.Gumz@medicine.ufl.edu.
1. Armstrong JA. Urinalysis in Western culture: a brief history. Kidney Int. 2007;71(5):384-387.

2. Mills JN. Human circadian rhythms. Physiol Rev. 1966;46(1):128-171.

3. Mills JN, Stanbury SW. Intrinsic diurnal rhythm in urinary electrolyte output. JPhysiol. 1951;115(1):18p-19p.

4. Mills JN. Diurnal rhythm in urine flow. J Physiol. 1951;113(4):528-536.

5. Mills JN, Stanbury SW. Persistent 24-hour renal excretory rhythm on a 12-hour cycle of activity. JPhysiol. 1952;117(1):22-37.

6. Sehgal A. Physiology flies with time. Cell. 2017;171(6):1232-1235.

7. Rijo-Ferreira F, Takahashi JS. Genomics of circadian rhythms in health and disease. Genome Med. 2019;11(1):82.

8. Takahashi JS. Transcriptional architecture of the mammalian circadian clock. Nat Rev Genet. 2017;18(3):164-179.

9. Patke A, et al. Molecular mechanisms and physiological importance of circadian rhythms. Nat Rev Mol Cell Biol. 2020;21(2):67-84.

10. Crislip GR, et al. Recent advances in understanding the circadian clock in renal physiology. Curr Opin Physiol. 2018;5:38-44.

11. Zhang D, et al. Loss of circadian gene Bmal1 in the collecting duct lowers blood pressure in male, but not female, mice. Am J Physiol Renal Physiol. 2020;318(3):F710-F719.

12. Zuber AM, et al. Molecular clock is involved in predictive circadian adjustment of renal function. Proc Natl Acad Sci US A. 2009;106(38):16523-16528.

13. Tokonami $\mathrm{N}$, et al. Local renal circadian clocks control fluid-electrolyte homeostasis and BP. J Am Soc Nephrol. 2014;25(7):1430-1439.

14. Solocinski K, et al. Desoxycorticosterone pivalate-salt treatment leads to non-dipping hypertension in Per1 knockout mice. Acta Physiol (Oxf). 2017;220(1):72-82.

15. Wang Q, et al. Cardiac hypertrophy, low blood pressure, and low aldosterone levels in mice devoid of the three circadian PAR bZip transcription factors DBP, HLF, and TEF. Am JPhysiol Regul Integr Comp Physiol. 2010;299(4):R1013-R1019.

16. Yeung J, et al. Transcription factor activity rhythms and tissue-specific chromatin interactions explain circadian gene expression across organs. Genome Res. 2018;28(2):182-191.

17. Crislip GR, et al. Differences in renal BMAL1 contribution to $\mathrm{Na}^{+}$homeostasis and blood pressure control in male and female mice. Am J Physiol Renal Physiol. 2020;318(6):F1463-F1477.

18. Nikolaeva S, et al. Nephron-specific deletion of circadian clock gene Bmal1 alters the plasma and renal metabolome and impairs drug disposition. JAm Soc Nephrol. 2016;27(10):2997-3004.

19. Stow LR, et al. The circadian protein period 1 contributes to blood pressure control and coordinately regulates renal sodium transport genes. Hypertension. 2012;59(6):1151-1156.

20. Douma LG, et al. Renal Na handling defect associated with PER1-dependent non-dipping hypertension in male mice. Am J Physiol Renal Physiol. 2018;314(6):F1138-F1144.

21. Castagna A, et al. Circadian exosomal expression of renal thiazide-sensitive $\mathrm{NaCl}$ cotransporter (NCC) and prostasin in healthy individuals. Proteomics Clin Appl. 2015;9(5-6):623-629.

22. Gumz ML, et al. An integrated view of potassium homeostasis. N Engl JMed. 2015;373(18):1787-1788.

23. Pilorz V, et al. The role of the circadian clock system in physiology. Pflugers Arch. 2018;470(2):227-239.

24. Rishi MA, et al. Daylight saving time: an American Academy of Sleep Medicine position statement. J Clin Sleep Med. 2020;16(10):1781-1784.

25. Uhm JY, et al. The association between shift work and chronic kidney disease in manual labor workers using data from the Korea National Health and Nutrition Examination Survey (KNHANES 2011-2014). Ann Occup Environ Med. 2018;30:69.

26. Rahim A, et al. The associations of shift work, sleep quality, and incidence of hypertension in Ontario adults: a population-based study. Can J Cardiol. 2021;37(3):513-518.

27. Lee DW, et al. Long work hours and decreased glomerular filtration rate in the Korean working population. Occup Environ Med. 2020;77(10):699-705.

28. Gamboa Madeira S, et al. The impact of different types of shift work on blood pressure and hypertension: a systematic review and meta-analysis. Int J Environ Res Public Health. 2021;18(13):6738.

29. Del Pinto R, et al. Diagnostic and therapeutic approach to sleep disorders, high blood pressure and cardiovascular diseases: a consensus document by the Italian Society of Hypertension (SIIA). High Blood Press Cardiovasc Prev. 2021;28(2):85-102.

30. Woon PY, et al. Aryl hydrocarbon receptor nuclear translocator-like (BMAL1) is asso- ciated with susceptibility to hypertension and type 2 diabetes. Proc Natl Acad Sci US A. 2007;104(36):14412-14417.

31. Dashti HS, et al. Clock genes explain a large proportion of phenotypic variance in systolic blood pressure and this control is not modified by environmental temperature. Am J Hypertens. 2016;29(1):132-140.

32. Agarwal R, Light RP. Sleep and activity in chronic kidney disease: a longitudinal study. Clin J Am Soc Nephrol. 2011;6(6):1258-1265.

33. Hsu CY, et al. Disrupted circadian rhythm in rats with nephrectomy-induced chronic kidney disease. Life Sci. 2012;91(3-4):127-131.

34. Motohashi $\mathrm{H}$, et al. The circadian clock is disrupted in mice with adenine-induced tubulointerstitial nephropathy. Kidney Int. 2020;97(4):728-740.

35. Lurbe E, et al. Increase in nocturnal blood pressure and progression to microalbuminuria in type 1 diabetes. N Engl J Med. 2002;347(11):797-805.

36. Mojon A, et al. Comparison of ambulatory blood pressure parameters of hypertensive patients with and without chronic kidney disease. Chronobiol Int. 2013;30(1-2):145-158.

37. Agarwal R, Andersen MJ. Prognostic importance of ambulatory blood pressure recordings in patients with chronic kidney disease. Kidney Int. 2006;69(7):1175-1180.

38. Covic A, et al. Dynamics of the circadian blood pressure profiles after renal transplantation. Transplantation. 2005;80(9):1168-1173.

39. Minutolo R, et al. Changing the timing of antihypertensive therapy to reduce nocturnal blood pressure in CKD: an 8-week uncontrolled trial. Am J Kidney Dis. 2007;50(6):908-917.

40. Poulter NR, et al. Randomized crossover trial of the impact of morning or evening dosing of antihypertensive agents on 24-hour ambulatory blood pressure: the HARMONY Trial. Hypertension. 2018;72(4):870-873.

41. Rahman M, et al. A trial of 2 strategies to reduce nocturnal blood pressure in blacks with chronic kidney disease. Hypertension. 2013;61(1):82-88.

42. Hermida RC, et al. Bedtime dosing of antihypertensive medications reduces cardiovascular risk in CKD. J Am Soc Nephrol. 2011;22(12):2313-2321.

43. Brown TM, et al. Angiotensin II regulates the activity of mouse suprachiasmatic nuclei neurons. Neuroscience. 2008;154(2):839-847.

44. Jaeger C, et al. Aryl hydrocarbon receptor deficiency alters circadian and metabolic rhythmicity. J Biol Rhythms. 2017;32(2):109-120. 
45. Xu CX, et al. Disruption of CLOCK-BMAL1 transcriptional activity is responsible for aryl hydrocarbon receptor-mediated regulation of Period1 gene. Toxicol Sci. 2010;115(1):98-108.

46. Portale AA, et al. Dietary intake of phosphorus modulates the circadian rhythm in serum concentration of phosphorus. Implications for the renal production of 1,25-dihydroxyvitamin D. J Clin Invest. 1987;80(4):1147-1154.

47. Svajger BA, et al. Development of experimental chronic kidney disease and vascular calcification alters diurnal variation of phosphate and its hormonal regulators. Physiol Rep. 2020;8(21):e14626.

48. Jubiz W, et al. Circadian rhythm in serum parathyroid hormone concentration in human subjects: correlation with serum calcium, phosphate, albumin, and growth hormone levels. J Clin Invest. 1972;51(8):2040-2046.

49. Nordholm A, et al. Circadian rhythm of activin A and related parameters of mineral metabolism in normal and uremic rats. Pflugers Arch. 2019;471(8):1079-1094.

50. Wide L, et al. Circadian rhythm of erythropoietin in human serum. Br J Haematol. 1989;72(1):85-90.

51. McKee LC, et al. Circadian variation in reticulocyte counts and immuno-detectable erythropoietin titers. Proc Soc Exp Biol Med. 1974;145(4):1284-1287.

52. el-Hajj Fuleihan G, et al. The parathyroid hormone circadian rhythm is truly endogenous - a general clinical research center study. J Clin Endocrinol Metab. 1997;82(1):281-286.

53. Da Costa D, et al. Determinants of sleep quality in women with systemic lupus erythematosus. Arthritis Rheum. 2005;53(2):272-278.

54 . McKinley PS, et al. The contributions of disease activity, sleep patterns, and depression to fatigue in systemic lupus erythematosus. A proposed model. Arthritis Rheum. 1995;38(6):826-834.

55. Valencia-Flores $M$, et al. Objective and subjective sleep disturbances in patients with systemic lupus erythematosus. Arthritis Rheum. 1999;42(10):2189-2193.

56. Tench CM, et al. The prevalence and associations of fatigue in systemic lupus erythematosus. Rheumatology (Oxford). 2000;39(11):1249-1254.

57 . Abad VC, et al. Sleep and rheumatologic disorders. Sleep Med Rev. 2008;12(3):211-228.

58. Brunner HI, et al. Difference in disease features between childhood-onset and adult-onset systemic lupus erythematosus. Arthritis Rheum. 2008;58(2):556-562.

59. Almaani S, et al. Update on lupus nephritis. Clin J Am Soc Nephrol. 2017;12(5):825-835.

60. Madaio MP. The role of autoantibodies in the pathogenesis of lupus nephritis. Semin Nephrol. 1999;19(1):48-56.

61. Trouw LA, et al. Anti-C1q autoantibodies deposit in glomeruli but are only pathogenic in combination with glomerular C1q-containing immune complexes. J Clin Invest. 2004;114(5):679-688.

62. Kalaaji M, et al. Critical comparative analyses of anti-alpha-actinin and glomerulus-bound antibodies in human and murine lupus nephritis. Arthritis Rheum. 2006;54(3):914-926.

63. Hedberg A, et al. Chromatin as a target antigen in human and murine lupus nephritis. Arthritis Res
Ther. 2011;13(2):214.

64. Stojan G, Petri M. Anti-C1q in systemic lupus erythematosus. Lupus. 2016;25(8):873-877.

65 . Weening JJ, et al. The classification of glomerulonephritis in systemic lupus erythematosus revisited. JAm Soc Nephrol. 2004;15(2):241-250.

66. Maria NI, Davidson A. Protecting the kidney in systemic lupus erythematosus: from diagnosis to therapy. Nat Rev Rheumatol. 2020;16(5):255-267.

67. Lema GP, et al. Chemokine expression precedes inflammatory cell infiltration and chemokine receptor and cytokine expression during the initiation of murine lupus nephritis. J Am Soc Nephrol. 2001;12(7):1369-1382.

68. Barrat FJ, et al. Nucleic acids of mammalian origin can act as endogenous ligands for Toll-like receptors and may promote systemic lupus erythematosus. JExp Med. 2005;202(8):1131-1139.

69. Bethunaickan R, et al. Identification of stage-specific genes associated with lupus nephritis and response to remission induction in $(\mathrm{NZB} \times \mathrm{NZW})$ F1 and NZM2410 mice. Arthritis Rheumatol. 2014;66(8):2246-2258.

70. Chang A, et al. In situ B cell-mediated immune responses and tubulointerstitial inflammation in human lupus nephritis. JImmunol. 2011;186(3):1849-1860.

71. Winchester R, et al. Immunologic characteristics of intrarenal T cells: trafficking of expanded CD ${ }^{+} \mathrm{T}$ cell $\beta$-chain clonotypes in progressive lupus nephritis. Arthritis Rheum 2012;64(5):1589-1600.

72. Hill GS, et al. Predictive power of the second renal biopsy in lupus nephritis: significance of macrophages. Kidney Int. 2001;59(1):304-316

73. Schiffer L, et al. Activated renal macrophages are markers of disease onset and disease remission in lupus nephritis. JImmunol. 2008;180(3):1938-1947.

74. Bethunaickan R, et al. A unique hybrid renal mononuclear phagocyte activation phenotype in murine systemic lupus erythematosus nephritis. J Immunol. 2011;186(8):4994-5003.

75. Vina ER, et al. Correlates of sleep abnormalities in systemic lupus: a cross-sectional survey in an urban, academic center. JClin Rheumatol. 2013;19(1):7-13.

76. Chandrasekhara PK, et al. The prevalence and associations of sleep disturbances in patients with systemic lupus erythematosus. Mod Rheumatol. 2009;19(4):407-415.

77. Isenberg DA, et al. Variation in circulating immune complex levels with diet, exercise, and sleep: a comparison between normal controls and patients with systemic lupus erythematosus. Ann Rheum Dis. 1981;40(5):466-469.

78. Young KA, et al. Less than 7 hours of sleep per night is associated with transitioning to systemic lupus erythematosus. Lupus. 2018;27(9):1524-1531.

79. Alarcon-Segovia D, et al. Familial aggregation of systemic lupus erythematosus, rheumatoid arthritis, and other autoimmune diseases in 1,177 lupus patients from the GLADEL cohort. Arthritis Rheum. 2005;52(4):1138-1147.

80. Hochberg MC. Updating the American College of Rheumatology revised criteria for the classification of systemic lupus erythematosus. Arthritis Rheum. 1997;40(9):1725.

81. Imai Y, et al. Exogenous glucocorticoid eliminates or reverses circadian blood pressure varia- tions. J Hypertens. 1989;7(2):113-120.

82. Bollinger T, et al. Sleep-dependent activity of T cells and regulatory T cells. Clin Exp Immunol. 2009;155(2):231-238.

83. Miyara M, et al. Global natural regulatory T cell depletion in active systemic lupus erythematosus. J Immunol. 2005;175(12):8392-8400.

84. Liu MF, et al. Decreased CD $4^{+} \mathrm{CD} 25^{+} \mathrm{T}$ cells in peripheral blood of patients with systemic lupus erythematosus. Scand J Immunol. 2004;59(2):198-202

85. Chalmers SA, et al. Macrophage depletion ameliorates nephritis induced by pathogenic antibodies. J Autoimmun. 2015;57:42-52:S08968411(14)00171-1.

86. Bethunaickan R, et al. Analysis of renal mononuclear phagocytes in murine models of SLE. Methods Mol Biol. 2012;900:207-232.

87. Triantafyllopoulou A, et al. Proliferative lesions and metalloproteinase activity in murine lupus nephritis mediated by type I interferons and macrophages. Proc Natl Acad Sci US A. 2010;107(7):3012-3017

88. Timmons GA, et al. Innate rhythms: clocks at the center of monocyte and macrophage function. Front Immunol. 2020;11:1743.

89. Alexander RK, et al. Bmal1 integrates mitochondrial metabolism and macrophage activation. Elife. 2020;9:e54090.

90. Early JO, et al. Circadian clock protein BMAL1 regulates IL-1 $\beta$ in macrophages via NRF2. Proc Natl Acad Sci U S A. 2018;115(36):E8460-E8468.

91. Keller M, et al. A circadian clock in macrophages controls inflammatory immune responses. Proc Natl Acad Sci U S A. 2009;106(50):21407-21412.

92. Jiang T, et al. Nrf2 suppresses lupus nephritis through inhibition of oxidative injury and the NF-kB-mediated inflammatory response. Kidney Int. 2014;85(2):333-343.

93. Jing C, et al. Macrophage metabolic reprogramming presents a therapeutic target in lupus nephritis. Proc Natl Acad Sci U S A 2020;117(26):15160-15171.

94. Cao Q, et al. Circadian clock cryptochrome proteins regulate autoimmunity. Proc Natl Acad Sci US A. 2017;114(47):12548-12553.

95. Palma BD, et al. Effects of sleep deprivation on the development of autoimmune disease in an experimental model of systemic lupus erythematosus. Am J Physiol Regul Integr Comp Physiol. 2006;291(5):R1527-R1532

96. Mishra R, et al. Reversible dysregulation of rena circadian rhythm in lupus nephritis. Mol Med. 2021;27(1):99.

97. Zubair A, Frieri M. NF- $\kappa$ B and systemic lupus erythematosus: examining the link. J Nephrol. 2013;26(6):953-959.

98. Hong $\mathrm{HK}$, et al. Requirement for NF- $\mathrm{BB}$ in maintenance of molecular and behavioral circadian rhythms in mice. Genes Dev. 2018;32(21-22):1367-1379.

99. Maury E, et al. Circadian clock dysfunction in human omental fat links obesity to metabolic inflammation. Nat Commun. 2021;12(1):2388.

100.Park J, et al. Effect of night shift work on the control of hypertension and diabetes in workers taking medication. Ann Occup Environ Med. 2019;31:e27.

101.Douma LG, Gumz ML. Circadian clock-mediated 
regulation of blood pressure. Free Radic Biol Med. 2018;119:108-114.

102.Uzu T, et al. Sodium restriction shifts circadian rhythm of blood pressure from nondipper to dipper in essential hypertension. Circulation. 1997;96(6):1859-1862.

103. Fujii T, et al. Circadian rhythm of natriuresis is disturbed in nondipper type of essential hypertension. Am J Kidney Dis. 1999;33(1):29-35.

104.Zhang D, et al. Timing of food intake drives the circadian rhythm of blood pressure. Function (Oxf). 2021;2(1):zqaa034.

105. Hou T, et al. Time-restricted feeding protects the blood pressure circadian rhythm in diabetic mice. Proc Natl Acad Sci U S A. 2021;118(25):e2015873118.

106.Duan LQ, et al. The correlation between urinary sodium excretion and blood pressure in hospitalized adult patients with hypertension. Adv Ther. 2021;38(5):2302-2314

107. Del Giorno R, et al. Impaired daytime urinary sodium excretion impacts nighttime blood pressure and nocturnal dipping at older ages in the general population. Nutrients. 2020;12(7):2013.

108. Chou YH, Chen YM. Aging and renal disease: old questions for new challenges. Aging Dis. 2021;12(2):515-528.

109. Hommos MS, et al. Structural and functional changes in human kidneys with healthy aging. JAm Soc Nephrol. 2017;28(10):2838-2844.

110. Denic A, et al. Structural and functional changes with the aging kidney. Adv Chronic Kidney Dis. 2016;23(1):19-28.

111. Denic A, et al. The substantial loss of nephrons in healthy human kidneys with aging. J Am Soc Nephrol. 2017;28(1):313-320.

112. Hoy WE, et al. A stereological study of glomerular number and volume: preliminary findings in a multiracial study of kidneys at autopsy. Kidney Int Suppl. 2003;(83):S31-S37.

113. Epstein M. Aging and the kidney. J Am Soc Nephrol.1996;7(8):1106-1122.

114. Kremers WK, et al. Distinguishing age-related from disease-related glomerulosclerosis on kidney biopsy: the Aging Kidney Anatomy study. Nephrol Dial Transplant. 2015;30(12):2034-2039.

115. Rule AD, et al. The association between age and nephrosclerosis on renal biopsy among healthy adults. Ann Intern Med. 2010;152(9):561-567.

116. Chan KW, et al. Age-related glomerular sclerosis: baseline values in Hong Kong. Pathology. 1990;22(4):177-180.

117. Kaplan C, et al. Age-related incidence of sclerotic glomeruli in human kidneys. Am J Pathol. 1975;80(2):227-234.

118. Baba M, et al. Longitudinal study of the decline in renal function in healthy subjects. PLoS One. 2015;10(6):e0129036.

119. Poggio ED, et al. Demographic and clinical characteristics associated with glomerular filtration rates in living kidney donors. Kidney Int. 2009;75(10):1079-1087.

120.Sarnak MJ, et al. Serum C-reactive protein and leptin as predictors of kidney disease progression in the Modification of Diet in Renal Disease Study. Kidney Int. 2002;62(6):2208-2215.

121. Lindeman RD, et al. Longitudinal studies on the rate of decline in renal function with age. $J \mathrm{Am}$ Geriatr Soc. 1985;33(4):278-285.

122. Rowe JW, et al. The effect of age on creatinine clearance in men: a cross-sectional and longitudinal study. J Gerontol. 1976;31(2):155-163.

123. Fliser $\mathrm{D}$, et al. Renal function in the elderly: impact of hypertension and cardiac function. Kidney Int. 1997;51(4):1196-1204.

124. Hollenberg NK, et al. Senescence and the renal vasculature in normal man. Circ Res. 1974;34(3):309-316.

125. Whelton PK, et al. Sodium reduction and weight loss in the treatment of hypertension in older persons: a randomized controlled trial of nonpharmacologic interventions in the elderly (TONE). TONE Collaborative Research Group. JAMA. 1998;279(11):839-846.

126. Luft FC, et al. Effects of age on renal sodium homeostasis and its relevance to sodium sensitivity. Am J Med. 1987;82(1b):9-15.

127. Hood S, Amir S. The aging clock: circadian rhythms and later life.J Jlin Invest. 2017;127(2):437-446.

128. Kim JH, Duffy JF. Circadian rhythm sleepwake disorders in older adults. Sleep Med Clin. 2018;13(1):39-50.

129. Broms U, et al. Long-term consistency of diurnal-type preferences among men. Chronobiol Int. 2014;31(2):182-188.

130. Roenneberg T, et al. Epidemiology of the human circadian clock. Sleep Med Rev. 2007;11(6):429-438.

131. Kripke DF, et al. Circadian phase response curves to light in older and young women and men. J Circadian Rhythms. 2007;5:4.

132. Sherman B, et al. Age-related changes in the circadian rhythm of plasma cortisol in man. JClin Endocrinol Metab. 1985;61(3):439-443.

133. Van Cauter E, et al. Effects of gender and age on the levels and circadian rhythmicity of plasma cortisol. J Clin Endocrinol Metab. 1996;81(7):2468-2473.

134.Lobban MC, Tredre BE. Diurnal rhythms of renal excretion and of body temperature in aged subjects. J Physiol. 1967;188(2):48P-49P.

135. Kirkland JL, et al. Patterns of urine flow and electrolyte excretion in healthy elderly people. $\mathrm{Br} \mathrm{Med} \mathrm{J}$ (Clin Res Ed). 1983;287(6406):1665-1667.

136.van Kerrebroeck P, et al. The standardisation of terminology in nocturia: report from the Standardisation Sub-committee of the International Continence Society. Neurourol Urodyn. 2002;21(2):179-183.

137. Bower WF, et al. Nocturia as a marker of poor health: causal associations to inform care. Neurourol Urodyn. 2017;36(3):697-705.

138. Bosch JL, Weiss JP. The prevalence and causes of nocturia. JUrol. 2013;189(1 suppl):S86-S92.

139. Rowe JW, et al. The influence of age on the renal response to water deprivation in man. Nephron. 1976;17(4):270-278

140.Epstein M, Hollenberg NK. Age as a determinant of renal sodium conservation in normal man. J Lab Clin Med. 1976;87(3):411-417.

141. Kaye M. Aging, circadian weight change, and nocturia. Nephron Physiol. 2008;109(1):p11-p18.

142. Blanker MH, et al. Normal values and determinants of circadian urine production in older men: a population based study. J Urol. 2002;168(4 pt 1):1453-1457.

143. Graugaard-Jensen C, et al. Nocturia and circadian blood pressure profile in healthy elderly male volunteers. JUrol. 2006;176(3):1034-1039.

144. Bliwise DL, et al. Nocturia: a highly prevalent disorder with multifaceted consequences. Urology. 2019;133S:3-13.

145. Feldstein CA. Nocturia in arterial hypertension: a prevalent, underreported, and sometimes underestimated association. J Am Soc Hypertens. 2013;7(1):75-84.

146. Bower WF, et al. Questions to ask a patient with nocturia. Aust J Gen Pract. 2018;47(7):465-469.

147. Abbott KC, et al. Survival by time of day of hemodialysis: analysis of United States Renal Data System Dialysis Morbidity and Mortality Waves III/IV. Am J Kidney Dis. 2003;41(4):796-806.

148. Bliwise DL, et al. Survival by time of day of hemodialysis in an elderly cohort. JAMA. 2001;286(21):2690-2694.

149.Schmitt EE, et al. The renal molecular clock: broken by aging and restored by exercise. $A m \mathrm{~J}$ Physiol Renal Physiol. 2019;317(5):F1087-F1093.

150.Fraga MF, et al. Epigenetic differences arise during the lifetime of monozygotic twins. Proc Natl Acad Sci US A. 2005;102(30):10604-10609.

151. Hannemann J, et al. Light therapy improves diurnal blood pressure control in night shift workers via reduction of catecholamines: the EuRhythDia study. J Hypertens. 2021;39(8):1678-1688.

152. Sutton EF, et al. Early time-restricted feeding improves insulin sensitivity, blood pressure, and oxidative stress even without weight loss in men with prediabetes. Cell Metab. 2018;27(6):1212-1221.

153. Wilkinson MJ, et al. Ten-hour time-restricted eating reduces weight, blood pressure, and atherogenic lipids in patients with metabolic syndrome. Cell Metab. 2020;31(1):92-104.

154. Koch BC, et al. Effects of nocturnal hemodialysis on melatonin rhythm and sleep-wake behavior: an uncontrolled trial. Am J Kidney Dis. 2009;53(4):658-664.

155. Roumeliotis A, et al. Cardiovascular benefits of extended-time nocturnal hemodialysis. Curr Vasc Pharmacol. 2021;19(1):21-33.

156. Reitz CJ, et al. SR9009 administered for one day after myocardial ischemia-reperfusion prevents heart failure in mice by targeting the cardiac inflammasome. Commun Biol. 2019;2:353.

157. Sulli G, et al. Training the circadian clock, clocking the drugs, and drugging the clock to prevent, manage, and treat chronic diseases. Trends Phar macol Sci. 2018;39(9):812-827.

158. Russcher M, et al. An observational study on disturbed peripheral circadian rhythms in hemodialysis patients. Chronobiol Int. 2015;32(6):848-857.

159. Canales MT, et al. Clock gene expression is altered in veterans with sleep apnea. Physiol Genomics. 2019;51(3):77-82.

160.Steimer M, et al. Expression of HO1 and PER2 can predict the incidence of delirium in trauma patients with concomitant brain injury. Sci Rep. 2021;11(1):15388.

161. Michael AK, et al. Cytosolic BMAL1 moonlights as a translation factor. Trends Biochem Sci. 2015;40(9):489-490. 